\title{
EDITORIAL
}

\section{Cystic fibrosis: to ion transport and beyond}

\author{
A. Bush and J. Davies
}

T he measurement of sweat electrolytes successfully diagnoses all but a tiny minority of patients with cystic fibrosis $(\mathrm{CF})$. The discovery of a gene for $\mathrm{CF}$, encoding the cystic fibrosis transmembrane conductance regulator (CFTR), has led to the understanding that CFTR is a chloride channel (hence the utility of the measurement of sweat electrolytes) and also regulates other ion channels, in particular the sodium transporter, ENaC. Measurement of transepithelial potential differences suggested that $\mathrm{ENaC}$ overactivity is pivotal in CF lung disease [1]. The CF mouse was found to have a poor lower airway phenotype for human CF lung disease, but the $\mathrm{ENaC} \beta$-subunit overexpressing mouse was a much better model for the devastating human CF lower airway disease [2]. Indeed, CFTR dysfunction, perhaps induced by tobacco smoke exposure, has been implicated in chronic obstructive pulmonary disease (COPD) [3]. After some controversy, a mechanism linking ion transport to $\mathrm{CF}$ lung disease was proposed [4]; the airway surface liquid (ASL) height was found to be equal in CF and normal airway cell monolayers if they were subjected to phasic contraction and relaxation, but in the presence of infection with respiratory syncytial virus, CF monolayers had reduced ASL height, and thus impaired mucociliary clearance [5], presumably accounting for the susceptibility to airway infection, inflammation and ultimately death by respiratory failure. So, CF and spin-off CFTR diseases, such as COPD are diseases of ion transport.

If not wrong, this view is perhaps over-simplistic. CFTR is now known to be much more than just an ion channel, a hole in the cell membrane with a bath plug-like structure to block it. CFTR has numerous other functions and a multiplicity of interactions with other proteins [6-8]. It is probably naïve to believe that absence or dysfunction of CFTR only affects ion transport. The model that CFTR dysfunction leads to ion transport problems which lead to infection, inflammation and eventual lung destruction, has already been challenged. Prospective, longitudinal physiological studies have established that infants with $\mathrm{CF}$ show evidence of airflow obstruction, independent of any clinical evidence of respiratory infection [9, 10], and this persists into school age [11-13]. Bronchoscopic studies have shown that some aspects of structural airway disease, in particular reticular basement membrane thickening, are independent of infection and inflammation [14]. It has been suggested that CFTR dysfunction may have a direct effect on airway smooth muscle [15]. Studies in CF mice have shown

Imperial College London, and Royal Brompton Hospital, London UK.

CORRESPONDENCE: A. Bush, Dept of Paediatric Respiratory Medicine, Royal Brompton Hospital, Sydney Street, London, SW3 6NP, UK. E-mail: a.bush@rbh.nthames.nhs.uk that there is thickening of the nasal respiratory epithelium and aggregations of lymphoid tissue in the CF mouse nose, even in the absence of any evidence of infection or excessive inflammation [16]. Taken together, these studies suggest there is a component of CF airway disease that is independent of downstream infection and inflammation, and which is very difficult to tie in to deficits in ion transport.

In this issue of the European Respiratory Journal, Dif et al. [17] report data that confirms this view. Their starting point was that CF mice had increased mucus production and MUC5AC expression (although it should be noted that this point is controversial) [18]. They performed an elegant series of experiments which convincingly show that cytosolic phospholipase A2 $\alpha$ (cPLA2 $\alpha$ ) interacts with CFTR to modulate mucus production, which, critically, is independent of chloride channel function. Thus $\mathrm{CFTR}^{-/-}$mice had increased mucus production and MUC5AC expression, exacerbated by Pseudomonas aeruginosa infection, but present even in uninfected mice. They showed that mucus production was induced by instillation of cPLA2 $\alpha$, and abolished in the absence of cPLA2 $\alpha$ activity, whether due to pharmacological inhibition or by cPLA2 $\alpha$ null mutation. Reduction of CFTR expression led to increased CPLA2 $\alpha$ activity and, as a downstream effect, a secondary increase in MUC5AC expression. Crucially, cPLA2 $\alpha$ appears to have no relevance whatsoever to the excessive neutrophilic inflammation characteristic of the $\mathrm{CF}$ airway. Furthermore, the CFTR chloride transport inhibitor CFTR $_{\text {inh }}-172$ did not lead to an increase in MUC5AC expression, underscoring the dissociation of chloride transport activity from airway mucus production.

Further evidence suggesting there is more to airway disease than just ion transport has come from the recent study of the peroxisome proliferator-activated receptor- $\gamma$ (PPAR- $\gamma$ ) pathway [19]. These investigators report data that also clearly dissociate abnormal chloride and sodium ion transport from the increased mucus production so characteristic of $\mathrm{CF}$ (originally called "mucoviscidosis"). The binding of the lipid 15-keto-prostaglandin-2 (15-keto-PGE 2 ) to PPAR- $\gamma$ regulates mucus production, and lipidomic studies suggest that dysregulation of this pathway due to reduced 15-keto-PGE 2 (amongst other lipids) may underlie the excessive mucus production in the airways and colons of $\mathrm{CFTR}^{-/-}$mice. The synthetic PPAR- $\gamma$ antagonist rosiglitazone reversed around a quarter of the genes up- and down-regulated in $\mathrm{CFTR}^{-/-}$mice compared with wild type. Cells treated with $\mathrm{CFTR}_{\text {inh }}-172$, or derived from $\mathrm{CFTR}^{-/-}$mice, showed the expected defect in chloride transport, but this was not reversed by roziglitazone. What roziglitazone did do was increase bicarbonate secretion via the genes encoding carbonic anhydrases 4 and 6 . Unlike the 
findings in the cPLA2 $\alpha$ pathway, PPAR $-\gamma$ is implicated in inflammation; PPAR- $\gamma$ agonists reduce the release of proinflammatory cytokines from airway epithelial cells infected with $P$. aeruginosa [20]. Taken together, these two manuscripts clearly establish that CFTR dysfunction exerts adverse effects on the airway independent of sodium and chloride transport. It is probably unsurprising that a multifunctional molecule as complex as CFTR, and an airway disease so multifaceted as that seen in $\mathrm{CF}$, do not bear a simple relationship such as "function $X$ causes disease $Y^{\prime}$.

Does this mean that efforts to correct ion transport are doomed to be fruitless, and that the use of ion transport as an end-point in CF clinical trials is a waste of time? This would be a radical, and almost certainly incorrect, conclusion. The evidence for the involvement of defective ion transport, in particular ENaC overactivity, in CF lung disease is compelling. Correction of ion transport defects is likely to bring substantial benefits. But before we assume that death from CF lung disease will itself be dead and buried when ion transport is normalised, we should remember that the other functions of CFTR may also need attention; correcting one CFTR function does not mean that everything in the garden is lovely. Indeed, this has been seen in gene therapy trials, in which some correction of chloride transport has been achieved, but correcting sodium hyperabsorption remains uniformly elusive. In the future, correction of aspects of CFTR dysfunction, by gene or other specific molecular therapies, may well lead to CF airway disease ceasing to be a killer of young adults. However, residual CFTR dysfunction may become a factor predisposing to early COPD in the nonsmoker, so there is no excuse for complacency.

\section{STATEMENT OF INTEREST}

None declared.

\section{REFERENCES}

1 Knowles M, Gatzy J, Boucher R. Increased bioelectric potential difference across respiratory epithelia in cystic fibrosis. $N$ Engl J Med 1981; 305: 1489-1495.

2 Mall M, Grubb BR, Harkema JR, et al. Increased airway epithelial $\mathrm{Na}^{+}$absorption produces cystic fibrosis-like lung disease in mice. Nat Med 2004; 10: 487-493.

3 Cantin AM, Hanrahan JW, Bilodeau G, et al. Cystic fibrosis transmembrane conductance regulator function is suppressed in cigarette smokers. Am J Respir Crit Care Med 2006; 173: 1139-1144.
4 Matsui H, Grubb BR, Tarran R, et al. Evidence for periciliary liquid layer depletion, not abnormal ion composition, in the pathogenesis of cystic fibrosis airways disease. Cell 1998; 95: 1005-1015.

5 Tarran R, Button B, Picher M, et al. Normal and cystic fibrosis airway surface liquid homeostasis. The effects of phasic shear stress and viral infections. J Biol Chem 2005; 280: 35751-35759.

6 Widdicombe JH. Yet another role for the cystic fibrosis transmembrane conductance regulator. Am J Respir Cell Mol Biol 2000; 22: 11-14.

7 Mehta A. CFTR: more than just a chloride channel. Pediatr Pulmonol 2005; 39: 292-298.

8 Wang X, Venable J, LaPointe P, et al. Hsp90 cochaperone Aha1 downregulation rescues misfolding of CFTR in cystic fibrosis. Cell 2006; 127: 803-815.

9 Ranganathan S, Dezateux C, Bush A, et al. Airway function in infants newly diagnosed with cystic fibrosis. Lancet 2001; 358: 1964-1965.

10 Ranganathan S, Bush A, Dezateux C, et al. Relative ability of full and partial forced expiratory manoeuvres to identify diminished airway function in infants with cystic fibrosis. Am J Respir Crit Care Med 2002; 166: 1350-1357.

11 Ranganathan SC, Stocks J, Dezateux C, et al. The evolution of airway function in early childhood following clinical diagnosis of cystic fibrosis. Am J Respir Crit Care Med 2004; 169: 928-933.

12 Kozlowska WJ, Bush A, Wade A, et al. Lung function from infancy to the preschool years following clinical diagnosis of cystic fibrosis. Am J Respir Crit Care Med 2008; 178: 42-49.

13 Nielsen KG, Pressler T, Klug B, et al. Serial lung function and responsiveness in cystic fibrosis during early childhood. Am J Respir Crit Care Med 2004; 169: 1209-1216.

14 Hilliard TN, Regamey N, Shute J, et al. Airway remodelling in children with cystic fibrosis. Thorax 2007; 62: 1074-1080.

15 Sutcliffe AM, Knox AJ. Muscling into cystic fibrosis airways. Thorax 2005; 60: 181-182.

16 Hilliard TN, Zhu J, Farley R, et al. Nasal abnormalities in cystic fibrosis mice independent of infection and inflammation. Am J Respir Cell Mol Biol 2008; 39: 19-25.

17 Dif F, Wu Y-Z, Burgel P-R, et al. Critical role of cytosolic phospholipase A2 $\alpha$ in bronchial mucus hypersecretion in CFTRdeficient mice. Eur Respir J 2010; 36: 1120-1130.

18 Henke MO, Renner A, Huber RM, et al. MUC5AC and MUC5B mucins are decreased in cystic fibrosis airway secretions. Am J Respir Cell Mol Biol 2004; 31: 86-91.

19 Harmon GS, Dumlao DS, Ng DT, et al. Pharmacological correction of a defect in PPAR- $\gamma$ signalling ameliorates disease severity in Cftr-deficient mice. Nat Med 2010; 16: 313-318.

20 Perez A, van Heeckeren AM, Nichols D, et al. Peroxisome proliferator-activated receptor- $\gamma$ in cystic fibrosis lung epithelium. Am J Physiol Lung Cell Mol Physiol 2008; 295: L303-L318. 